DOI 10.1007/s10958-020-04981-0

Journal of Mathematical Sciences, Vol. 249, No. 6, September, 2020

\title{
DISTRIBUTIONS SUPPORTED ON CONICAL SURFACES AND GENERATED CONVOLUTIONS
}

\author{
V. B. Vasil'ev \\ Belgorod National Research University \\ 14/1 Studencheskaya St., Belgorod 308007, Russia \\ vbv57@inbox.ru
}

UDC 517.983

\begin{abstract}
We describe the structure of distributions supported on conical surfaces and calculate the Fourier transform for some cones. The results are represented as convolutions with particular kernels. We use transmutation operators owing to which it is possible to clarify connections between the change of variables for a distribution and its Fourier transform. Bibliography: 17 titles.
\end{abstract}

A lot of examples of distributions supported on various type surfaces in $m$-dimensional spaces can be found in $[1,2]$. However, in the literature, there are no results concerning distributions in the general form (counterparts of the Schwartz theorem on the general form of a distribution supported at a point in the one-dimensional case [3]). This paper is motivated by the recent results of [4]-[8], where pseudodifferential equations are studied in domains with conical points on the boundary in the multidimensional $(m \geqslant 3)$ case.

\section{Distributions and Change of Variables}

1.1. Choice of test functions. Let $C$ be an acute convex cone in $\mathbb{R}^{m}$ containing no entire line. Assume that the conical surface is given by the equation $x_{m}=\varphi\left(x^{\prime}\right), x^{\prime}=\left(x_{1}, \ldots, x_{m-1}\right)$, where $\varphi: \mathbb{R}^{m-1} \rightarrow \mathbb{R}$ is a smooth function on $\mathbb{R}^{m-1} \backslash\{0\}$ such that $\varphi(0)=0$. We introduce the change of variables $t_{1}=x_{1}, t_{2}=x_{2}, \ldots, t_{m-1}=x_{m-1}, t_{m}=x_{m}-\varphi\left(x^{\prime}\right)$ and denote by $T_{\varphi}$ : $\mathbf{R}^{m} \rightarrow \mathbf{R}^{m}$ the change operator. It is obvious that this transformation is smooth except for the origin. We introduce the change of variables for the following class of distributions. For the space of test functions we take the Lizorkin space $\Phi\left(\mathbb{R}^{m}\right)[9]$ which is a subspace of the Schwartz space $S\left(\mathbb{R}^{m}\right)$ of infinitely differentiable functions that are rapidly decreasing at infinity and vanish at the origin, together with all its derivatives. If $\Phi^{\prime}\left(\mathbb{R}^{m}\right)$ and $S^{\prime}\left(\mathbb{R}^{m}\right)$ denote the corresponding spaces of distributions, then $\Phi^{\prime}\left(\mathbb{R}^{m}\right) \supset S^{\prime}\left(\mathbb{R}^{m}\right)$ and all operations with distributions in $\Phi^{\prime}\left(\mathbb{R}^{m}\right)$ are legitimate for distributions in $S^{\prime}\left(\mathbb{R}^{m}\right)$.

Translated from Problemy Matematicheskogo Analiza 103, 2020, pp. 63-70.

1072-3374/20/2496-0885 (C) 2020 Springer Science+Business Media, LLC 
Definition 1.1. The variable change operator on functions in $\Phi\left(\mathbb{R}^{m}\right)$ is defined by

$$
\left(\mathrm{T}_{\varphi} \psi\right)(x)=\psi\left(T_{\varphi} x\right)
$$

It is easy to see that the Jacobian of $T_{\varphi}$ is everywhere (except for 0 ) equal to 1 . By the choice of the class of test functions, we can ignore the point 0 . It is clear that the operator $\mathrm{T}_{\varphi}$ is invertible and its inverse is defined by $\left(\mathrm{T}_{\varphi}^{-1} \psi\right)(x)=\psi\left(T_{\varphi}^{-1} x\right)$.

Let $f$ be a locally integrable function generating a distribution by

$$
(f, \psi)=\int_{\mathbb{R}^{m}} f(x) \psi(x) d x
$$

The functional $\mathbf{T}_{\varphi} f$ is defined by $\left(\mathbf{T}_{\varphi} f, \psi\right)=\left(f, \mathrm{~T}_{\varphi}^{-1} \psi\right)$ since

$$
\left(\mathrm{T}_{\varphi} f, \psi\right)=\int_{\mathbb{R}^{m}}\left(\mathrm{~T}_{\varphi} f\right)(x) \psi(x) d x \equiv \int_{\mathbb{R}^{m}} f\left(T_{\varphi} x\right) \psi(x) d x=\int_{\mathbb{R}^{m}} f(x) \psi\left(T_{\varphi}^{-1} x\right) d x \equiv\left(f, T_{\varphi}^{-1} \psi\right)
$$

We use the following result based on the Schwartz theorem about a one-dimensional distribution in $S^{\prime}(\mathbb{R})$ supported at the point $0[2,3]$.

Proposition 1.1. If a distribution $f \in S^{\prime}\left(\mathbb{R}^{m}\right)$ is supported on the hyperplane $x_{m}=0$, then

$$
f(x)=\sum_{k=0}^{n} c_{k}\left(x^{\prime}\right) \otimes \delta^{(k)}\left(x_{m}\right), \quad x=\left(x^{\prime}, x_{m}\right),
$$

where $c_{k} \in S^{\prime}\left(\mathbb{R}^{m-1}\right), k=0,1, \ldots, n$, are arbitrary distributions.

\subsection{Change of variables for distributions.}

Definition 1.2. The operator $\mathbf{T}_{\varphi}$ acts by the formula

$$
\left(\mathbf{T}_{\varphi} f, \psi\right)=\left(f, \mathrm{~T}_{\varphi}^{-1} \psi\right) \quad \forall \psi \in S\left(\mathbb{R}^{m}\right) .
$$

Proposition 1.2. If a distribution $f \in S^{\prime}\left(\mathbb{R}^{m}\right)$ is supported on $\partial C$, then $\mathbf{T}_{\varphi} f$ is supported on $\mathbb{R}^{m-1}$.

Propositions 1.1 and 1.2 imply the following assertion.

Theorem 1.1. Any distribution $f \in S^{\prime}\left(\mathbb{R}^{m}\right)$ supported on the conical surface $\partial C$ can be written as

$$
f(x)=\mathbf{T}_{\varphi}^{-1}\left(\sum_{k=0}^{n} c_{k}\left(y^{\prime}\right) \otimes \delta^{(k)}\left(y_{m}\right)\right)
$$

where $c_{k} \in S^{\prime}\left(\mathbb{R}^{m-1}\right), k=0,1, \ldots, n$, are arbitrary distributions.

Theorem 1.1 in this setting does not yield much information, but we need a version of this theorem formulated in terms of Fourier images. 


\section{Fourier Transform of Distributions}

It is natural to ask how the transform of a distribution supported on a conical surface looks like. Thus, the Fourier transform of the distribution (1.1) is a polynomial of degree $n$ in the variable $\xi_{m}$ with coefficients $\widetilde{c}_{k}\left(\xi^{\prime}\right), k=0,1, \ldots, n$.

For $u(x) \in S\left(\mathbb{R}^{m}\right)$ the Fourier transform is defined by [1]-[3]

$$
(F u)(\xi) \equiv \widetilde{u}(\xi)=\int_{\mathbb{R}^{m}} e^{i x \cdot \xi} u(x) d x .
$$

The Fourier transform of a distribution is defined by the formula $(F f, \psi)=(f, F \psi)$, and, consequently, $\left(F \mathrm{~T}_{\varphi} f, \varphi\right)=\left(f, \mathrm{~T}_{\varphi}^{-1} F \varphi\right)$.

By the above properties, to establish connections between $\mathrm{T}_{\varphi}$ and the Fourier transform, it suffices to consider test functions.

Let $f \in S^{\prime}\left(\mathbb{R}^{m}\right)$ be supported on the conical surface $\partial C$. By Theorem 1.1, it has the form (1.2). We obtain the following chain of identities:

$$
\begin{aligned}
(F f, \psi) & =\left(F \mathbf{T}_{\varphi}^{-1}\left(\sum_{k=0}^{n} c_{k}\left(y^{\prime}\right) \otimes \delta^{(k)}\left(y_{m}\right)\right), \psi\right) \\
& =\left(F \mathbf{T}_{\varphi}^{-1} F^{-1} F\left(\sum_{k=0}^{n} c_{k}\left(y^{\prime}\right) \otimes \delta^{(k)}\left(y_{m}\right)\right), \psi\right)=\left(V_{\varphi}\left(\sum_{k=0}^{n} \widetilde{c}_{k}\left(\xi^{\prime}\right) \xi_{m}^{k}\right), \psi\right),
\end{aligned}
$$

where $F \mathbf{T}_{\varphi}^{-1} F^{-1} \equiv V_{\varphi}$ denotes the operator defined originally on $S\left(\mathbb{R}^{m}\right)$. We note that this operator is invertible; moreover, $V_{\varphi}^{-1}=F \mathbf{T}_{\varphi}^{-1} F^{-1}$ and $\mathbf{T}_{\varphi}^{-1}=\mathbf{T}_{-\varphi}$. By the above observations, we can conclude that

$$
F f=V_{\varphi}\left(\sum_{k=0}^{n} \widetilde{c}_{k}\left(\xi^{\prime}\right) \xi_{m}^{k}\right) \text {. }
$$

2.1. Kernel of the operator $V_{\varphi}$. Based on the key identity $F \mathbf{T}_{\varphi}^{-1}=V_{\varphi} F$, we conclude that it is more convenient to work with the operator $V_{\varphi}$ in the Fourier image. We begin with the left-hand side. We fix $u \in S\left(\mathbb{R}^{m}\right)$ and calculate

$$
\begin{aligned}
\left(F \mathrm{~T}_{\varphi}^{-1} u\right)(\xi) & =\int_{\mathbb{R}^{m}} e^{i y \cdot \xi}\left(\mathrm{T}_{\varphi}^{-1} u\right)(y) d y=\int_{\mathbb{R}^{m}} e^{i y \cdot \xi^{\prime}} u\left(y^{\prime}, y_{m}+\varphi\left(y^{\prime}\right)\right) d y \\
& =\int_{\mathbb{R}^{m}} e^{i x \cdot \xi} e^{-i \xi_{m} \varphi\left(x^{\prime}\right)} u\left(x^{\prime}, x_{m}\right) d x^{\prime} d x_{m}=\int_{\mathbb{R}^{m}} e^{i x^{\prime} \cdot \xi^{\prime}} e^{-i \xi_{m} \varphi\left(x^{\prime}\right)} \widehat{u}\left(x^{\prime}, \xi_{m}\right) d x^{\prime},
\end{aligned}
$$

where $\widehat{u}\left(x^{\prime}, \xi_{m}\right)$ denotes the Fourier transform of $u\left(x^{\prime}, x_{m}\right)$ with respect to $x_{m}$. Taking into account the properties of the Fourier transform, we can make the following conclusions.

We denote $F_{x^{\prime} \rightarrow \xi^{\prime}}\left(e^{-i \xi_{m} \varphi\left(x^{\prime}\right)}\right) \equiv K_{\varphi}\left(\xi^{\prime}, \xi_{m}\right)$. Then we find the integral representation of the operator $V_{\varphi}$ :

$$
\left(F \mathrm{~T}_{\varphi}^{-1} u\right)(\xi)=\int_{\mathbb{R}^{m}} K_{\varphi}\left(\xi^{\prime}-\eta^{\prime}, \xi_{m}\right) \widetilde{u}\left(\eta^{\prime}, \xi_{m}\right) d \eta^{\prime}
$$

where $K\left(\xi^{\prime}, \xi_{m}\right)$ is the Fourier image of the corresponding distributions. 
2.2. Plane case: exact construction of $V_{\varphi}$. In the case $m=2$, only one cone is of interest for us; namely, $C_{+}^{a}=\left\{x \in \mathbb{R}^{2}: x=\left(x_{1}, x_{2}\right), x_{2}>a\left|x_{1}\right|, a>0\right\}$. We calculate

$$
\begin{aligned}
& \left(F_{\varphi}^{-1} u\right)(\xi)=\int_{-\infty}^{+\infty} e^{-i a\left|y_{1}\right| \xi_{2}} e^{-i y_{1} \xi_{1}} \widehat{u}\left(y_{1}, \xi_{2}\right) d y_{1} \\
& =\int_{-\infty}^{+\infty} \chi_{+}\left(y_{1}\right) e^{-i a y_{1} \xi_{2}} e^{-i y_{1} \xi_{1}} \widehat{u}\left(y_{1}, \xi_{2}\right) d y_{1}+\int_{-\infty}^{+\infty} \chi_{-}\left(y_{1}\right) e^{-i a y_{1} \xi_{2}} e^{i y_{1} \xi_{1}} \widehat{u}\left(y_{1}, \xi_{2}\right) d y_{1} \\
& =\int_{-\infty}^{+\infty} \chi_{+}\left(y_{1}\right) e^{-i y_{1}\left(a \xi_{2}+\xi_{1}\right)} \widehat{u}\left(y_{1}, \xi_{2}\right) d y_{1}+\int_{-\infty}^{+\infty} \chi_{-}\left(y_{1}\right) e^{-i y_{1}\left(a \xi_{2}-\xi_{1}\right)} \widehat{u}\left(y_{1}, \xi_{2}\right) d y_{1},
\end{aligned}
$$

where $\widehat{u}\left(y_{1}, \xi_{2}\right)$ denotes the one-dimensional Fourier transform with respect to the second variable. The last two terms are the Fourier transforms of the functions

$$
\chi_{+}\left(y_{1}\right) e^{-i y_{1}\left(a \xi_{2}+\xi_{1}\right)} \widehat{u}\left(y_{1}, \xi_{2}\right), \quad \chi_{+}\left(y_{1}\right) e^{-i y_{1}\left(a \xi_{2}+\xi_{1}\right)} \widehat{u}\left(y_{1}, \xi_{2}\right)
$$

with respect to $y_{1}$, so that we can use the following properties [10] (the Sokhotski formula $[11,12]$, written for one variable):

$$
\begin{aligned}
& \int_{-\infty}^{+\infty} \chi_{+}(x) e^{-i x \xi} u(x) d x=\frac{1}{2} \widetilde{u}(\xi)+v \cdot p \cdot \frac{i}{2 \pi} \int_{-\infty}^{+\infty} \frac{\widetilde{u}(\eta) d \eta}{\xi-\eta}, \\
& \int_{-\infty}^{+\infty} \chi_{-}(x) e^{-i x \xi} u(x) d x=\frac{1}{2} \widetilde{u}(\xi)-v \cdot p \cdot \frac{i}{2 \pi} \int_{-\infty}^{+\infty} \frac{\widetilde{u}(\eta) d \eta}{\xi-\eta} .
\end{aligned}
$$

Taking into account these properties, we conclude that

$$
\begin{aligned}
\left(F \mathrm{~T}_{\varphi}^{-1} u\right)(\xi) & =\frac{\widetilde{u}\left(a \xi_{2}+\xi_{1}, \xi_{2}\right)+\widetilde{u}\left(a \xi_{2}-\xi_{1}, \xi_{2}\right)}{2} \\
& +v \cdot p \cdot \frac{i}{2 \pi} \int_{-\infty}^{+\infty} \frac{\widetilde{u}\left(\eta, \xi_{2}\right) d \eta}{a \xi_{2}+\xi_{1}-\eta}-v \cdot p \cdot \frac{i}{2 \pi} \int_{-\infty}^{+\infty} \frac{\widetilde{u}\left(\eta, \xi_{2}\right) d \eta}{a \xi_{2}-\xi_{1}-\eta} \equiv\left(V_{\varphi} \widetilde{u}\right)(\xi) .
\end{aligned}
$$

Theorem 2.1. In the case of an plane angle $C_{+}^{a}$, the operator $V_{\varphi}$ has the form

$$
\begin{aligned}
\left(V_{\varphi} \widetilde{u}\right)(\xi) & =\frac{\widetilde{u}\left(a \xi_{2}+\xi_{1}, \xi_{2}\right)+\widetilde{u}\left(a \xi_{2}-\xi_{1}, \xi_{2}\right)}{2} \\
& +v \cdot p \cdot \frac{i}{2 \pi} \int_{-\infty}^{+\infty} \frac{\widetilde{u}\left(\eta, \xi_{2}\right) d \eta}{a \xi_{2}+\xi_{1}-\eta}-v \cdot p \cdot \frac{i}{2 \pi} \int_{-\infty}^{+\infty} \frac{\widetilde{u}\left(\eta, \xi_{2}\right) d \eta}{a \xi_{2}-\xi_{1}-\eta}
\end{aligned}
$$

2.3. Multidimensional case: construction of kernel $K_{\varphi}$. It turns out that the kernel $K_{\varphi}$ is "computable" in the particular case $\varphi\left(x^{\prime}\right)$. We set $\varphi\left(x^{\prime}\right)=a\left|x^{\prime}\right|, a>0$, and use formulas 
from [13] (cf. also [14], where real counterparts of these formulas are given in the form of the Poisson kernel)

$$
K_{\varphi}\left(\xi^{\prime}, \xi_{m}\right)=\frac{i \xi_{m} a 2^{m-1} \pi^{\frac{m-2}{2}} \Gamma(m / 2)}{\left(\xi_{1}^{2}+\xi_{2}^{2}+\ldots+\xi_{m-1}^{2}-a^{2} \xi_{m}^{2}\right)^{m / 2}} .
$$

Consequently, for our multidimensional cone the operator $V_{\varphi}$ has the form

$$
\left(V_{\varphi} \widetilde{u}\right)(\xi)=\lim _{\tau \rightarrow 0+} \int_{\mathbb{R}^{m-1}} \frac{i z_{m} a 2^{m-1} \pi^{\frac{m-2}{2}} \Gamma(m / 2) \widetilde{u}\left(\eta^{\prime}, \xi_{m}\right) d \eta^{\prime}}{\left(\left(\xi_{1}-\eta_{1}\right)^{2}+\left(\xi_{2}-\eta_{2}\right)^{2}+\ldots+\left(\xi_{m-1}-\eta_{m-1}\right)^{2}-a^{2} z_{m}^{2}\right)^{m / 2}},
$$

where $z_{m}=\xi_{m}-i \tau, \tau>0$, which gives us a reason to call it the conical potential.

Theorem 2.2. For a multidimensional circular cone the operator $V_{\varphi}$ has the form (2.1).

Remark 2.1. Formula (2.1) should be understood in the sense of the theory of distributions. Exact definitions will be given below for the operator $V_{\varphi}$ in the space $S^{\prime}\left(\mathbb{R}^{m}\right)$.

We consider a cone in the multidimensional space

$$
\left\{x \in \mathbb{R}^{m}: x_{m}>\sum_{k=1}^{m-1} a_{k}\left|x_{k}\right|, a_{k}>0\right\} .
$$

Then the operator $V_{\varphi}$ is also computable (cf. [15] in the case $m=3$ ).

Finally, taking into account the above calculations, we can give a definition of the operator $V_{\varphi}$ for distributions.

Definition 2.1. For distributions $f \in S^{\prime}\left(\mathbb{R}^{m}\right)$ we introduce the transform $V_{\varphi}$ by

$$
\left(V_{\varphi} \widetilde{f}, \psi\right) \equiv\left(\tilde{f}, V_{-\varphi} \psi\right) \quad \forall \psi \in S\left(\mathbb{R}^{m}\right) .
$$

\section{General Solution and Boundary Conditions}

3.1. Pseudodifferential equation and wave factorization of symbol. We consider the simplest cone $C_{+}^{a}=\left\{x \in \mathbb{R}^{m}: x=\left(x^{\prime}, x_{m}\right), x_{m}>a\left|x^{\prime}\right|, a>0\right\}$ and show the role of the above-mentioned distributions in the construction of solutions to the pseudodifferential equation

$$
\left(A u_{+}\right)(x)=f(x), \quad x \in C_{+}^{a},
$$

in the space $H^{s}\left(C_{+}^{a}\right)$ and the right-hand side $H_{0}^{s-\alpha}\left(C_{+}^{a}\right)$ under the following condition on the symbol $A(\xi)[4,6,16]$ :

$$
c_{1}(1+|\xi|)^{\alpha} \leqslant|A(\xi)| \leqslant c_{2}(1+|\xi|)^{\alpha}
$$

provided that the symbol admits the wave factorization with respect to $C_{+}^{a}[7,5]$

$$
A(\xi)=A_{\neq}(\xi) \cdot A_{=}(\xi)
$$

with index æ. The structure of solutions to Equation (3.1) is known in the case $æ-s=n+\delta$, $n \in \mathbb{N},|\delta|<1 / 2$ and takes the following form in the simplest case $n=1, f \equiv 0$ :

$$
\widetilde{u}_{+}(\xi)=A_{\neq}^{-1}(\xi) V_{-\varphi} \widetilde{c}_{1}\left(\xi^{\prime}\right)
$$


or, in more detail:

$$
\widetilde{u}_{+}(\xi)=A_{\neq}^{-1}(\xi) \int_{\mathbb{R}^{m-1}} \frac{i \xi_{m} a 2^{m-1} \pi^{\frac{m-2}{2}} \Gamma(m / 2) \widetilde{c}_{1}\left(\eta^{\prime}\right) d \eta^{\prime}}{\left(\left(\xi_{1}-\eta_{1}\right)^{2}+\left(\xi_{2}-\eta_{2}\right)^{2}+\ldots+\left(\xi_{m-1}-\eta_{m-1}\right)^{2}-a^{2} \xi_{m}^{2}\right)^{m / 2}},
$$

where $c_{1}$ is an arbitrary function in a suitable Sobolev-Slobodetski space [15].

3.2. Integral boundary condition. There is one arbitrary function in (3.2). If this function is fixed, then the solution becomes unique. To determine it in a unique way, we need to consider the equation with some additional (boundary) condition. As a result, we obtain a boundary value problem. In the simplest case, we obtain the problem with integral boundary condition

$$
\int_{0}^{+\infty} u_{+}\left(x^{\prime}, x_{m}\right) d x_{m}=g\left(x^{\prime}\right)
$$

which can be written in the Fourier images as

$$
\widetilde{u}_{+}\left(\xi^{\prime}, 0\right)=\widetilde{g}\left(\xi^{\prime}\right)
$$

Substituting (3.4) into (3.2), we obtain the following integral equation for $\widetilde{c}_{1}$ :

$$
\lim _{\xi_{m} \rightarrow 0} \int_{\mathbb{R}^{m-1}} \frac{i \xi_{m} a 2^{m-1} \pi^{\frac{m-2}{2}} \Gamma(m / 2) \widetilde{c}_{1}\left(\eta^{\prime}\right) d \eta^{\prime}}{\left(\left(\xi_{1}-\eta_{1}\right)^{2}+\left(\xi_{2}-\eta_{2}\right)^{2}+\ldots+\left(\xi_{m-1}-\eta_{m-1}\right)^{2}-a^{2} \xi_{m}^{2}\right)^{m / 2}}=A_{\neq}\left(\xi^{\prime}, 0\right) \widetilde{g}\left(\xi^{\prime}\right) .
$$

Since the right-hand side of (3.5) is known and the left-hand side is the convolution with the delta-function, we can apply the (inverse) Fourier transform. According to [1], we obtain the expression

$$
c_{1}\left(x^{\prime}\right)=\int_{\mathbb{R}^{m-1}} M\left(x^{\prime}-y^{\prime}\right) g\left(y^{\prime}\right) d y^{\prime},
$$

where $M\left(x^{\prime}\right)=F_{\xi^{\prime} \rightarrow x^{\prime}}^{-1} A_{\neq}\left(\xi^{\prime}, 0\right)$.

Thus, the function $c_{1}\left(x^{\prime}\right)$ is uniquely found by formula (3.6). Consequently, the problem (3.1), (3.3) has a unique solution.

3.3. The Dirichlet boundary condition. To impose the Dirichlet condition on the general solution $u_{+}$on the conical boundary $\partial C_{+}^{a}$ in a convenient form, we need to make some transformations. Applying the transform $\mathrm{T}_{\varphi}$ to the solution $u_{+}$, we obtain the function $\left(\mathrm{T}_{\varphi} u_{+}\right)(x)$ on $\mathbb{R}_{+}^{m}$. Thus, the condition

$$
\left(\mathrm{T}_{\varphi} u_{+}\right)\left(x^{\prime}, 0\right)=g\left(x^{\prime}\right)
$$

is the Dirichlet condition on $\partial C_{+}^{a}$. In the Fourier images, this condition has the form

$$
\int_{-\infty}^{+\infty}\left(F \mathrm{~T}_{\varphi} u_{+}\right)\left(\xi^{\prime}, \xi_{m}\right) d \xi_{m}=\widetilde{g}\left(\xi^{\prime}\right)=\int_{-\infty}^{+\infty}\left(V_{\varphi} \widetilde{u}_{+}\right)\left(\xi^{\prime}, \xi_{m}\right) d \xi_{m}
$$

where formula (2.1) was taken into account. Consequently, assuming the condition (3.7), we apply the operator $V_{\varphi}$ to both sides of (3.2) and then integrate with respect to $\xi_{m}$. Then we 
write the result as

$$
\begin{aligned}
\widetilde{g}\left(\xi^{\prime}\right) & =d_{m} \int_{\mathbb{R}^{m}} \frac{\left.\xi_{m} A_{\neq}^{-1}\left(\zeta^{\prime}, \xi_{m}\right)\right)}{\left(\left|\xi^{\prime}-\zeta^{\prime}\right|^{2}-a^{2} \xi_{m}^{2}\right)^{m / 2}}\left(\int_{\mathbb{R}^{m-1}} \frac{\xi_{m} \widetilde{c}_{1}\left(\eta^{\prime}\right) d \eta^{\prime}}{\left(\left|\zeta^{\prime}-\eta^{\prime}\right|^{2}-a^{2} \xi_{m}^{2}\right)^{m / 2}}\right) d \zeta^{\prime} d \xi_{m} \\
& =d_{m} \int_{\mathbb{R}^{m}} \frac{\left.\xi_{m} A_{\neq}^{-1}\left(\zeta^{\prime}, \xi_{m}\right)\right)}{\left(\left|\xi^{\prime}-\zeta^{\prime}\right|^{2}-a^{2} \xi_{m}^{2}\right)^{m / 2}} \widetilde{C}_{1}\left(\zeta^{\prime}, \xi_{m}\right) d \zeta^{\prime} d \xi_{m},
\end{aligned}
$$

where

$$
\widetilde{C}_{1}\left(\zeta^{\prime}, \xi_{m}\right)=\int_{\mathbb{R}^{m-1}} \frac{\xi_{m} \widetilde{c}_{1}\left(\eta^{\prime}\right) d \eta^{\prime}}{\left(\left|\zeta^{\prime}-\eta^{\prime}\right|^{2}-a^{2} \xi_{m}^{2}\right)^{m / 2}} .
$$

Thus, the main point of finding solutions is the method for solving Equation (3.8) for $\widetilde{C}_{1}\left(\zeta^{\prime}, \xi_{m}\right)$ since $c_{1}$ can be determined by the Fourier transform.

\section{Coboundary Operators and Solvability Conditions}

In this section, we show what to expect in the case $æ-s=n+\delta,-n \in \mathbb{N},|\delta|<1 / 2$, (cf. $[4,7,10,17])$. We consider an arbitrary convex cone $C$ containing no entire line and the equation

$$
\left(A u_{+}\right)(x)=f(x), \quad x \in C .
$$

If $f \in H_{0}^{s-\alpha}(C)$, then $f \in H_{0}^{æ-\delta-\alpha}(C)$ since $s-\alpha=æ-n-\delta-\alpha>æ-\delta-\alpha$. Then there exists a unique solution $w_{+} \in H^{æ-\delta}(C)$ Equation (4.1) such that

$$
\widetilde{w}_{+}(\xi)=A_{\neq}^{-1}(\xi) B_{m} A_{=}^{-1}(\xi) \widetilde{l f}(\xi),
$$

where $B_{m}$ is the operator generated by the Bochner kernel for the cone $C$ and $l f$ is an arbitrary extension of $f$ on $H^{s-\alpha}\left(\mathbb{R}^{m}\right)[7,5]$.

The difficulty is that formula (4.2) yields a solution in a wider space, and there is arbitrariness in the choice of a solution in the required class $H^{s}(C)$.

As above, we apply the transform operators to reduce (4.2) to the case of a half-space and use the corresponding results of [10]. We use [10] for the notation and facts related to the theory of one-dimensional singular integral equations and classical Riemann problem [11, 12].

For the operators originally defined on the Schwartz class $S\left(\mathbb{R}^{m}\right)$ of infinitely differentiable functions that are rapidly decreasing at infinity we introduce the notation

$$
\begin{aligned}
& \left(\Pi_{ \pm} \widetilde{u}\right)\left(\xi^{\prime}, \xi_{m}\right)= \pm \frac{i}{2 \pi} \lim _{\tau \rightarrow 0+} \int_{-\infty}^{+\infty} \frac{\widetilde{u}\left(\xi^{\prime}, \eta_{m}\right) d \eta_{m}}{\xi_{m} \pm i \tau-\eta_{m}} \\
& \left(\Pi^{\prime} \widetilde{u}\right)\left(\xi^{\prime}\right)=\int_{-\infty}^{+\infty} \widetilde{u}\left(\xi^{\prime}, \xi_{m}\right) d \xi_{m}
\end{aligned}
$$

$\Lambda_{ \pm}$is a pseudodifferential operator with symbol $\Lambda_{ \pm}\left(\xi^{\prime}, \xi_{m}\right)=\xi_{m} \pm i\left|\xi^{\prime}\right| \pm i$. In this case, for $\widetilde{u}_{+} \in \widetilde{H}^{n+\delta}\left(\mathbb{R}^{m}\right), n \in \mathbb{N},|\delta|<1 / 2$, we have the decomposition [10]

$$
\left(\Pi_{+} \widetilde{u}\right)\left(\xi^{\prime}, \xi_{m}\right)=\sum_{j=1}^{k} \frac{i\left(\Pi^{\prime} \Lambda_{+}^{j-1} \widetilde{u}\right)\left(\xi^{\prime}\right)}{\Lambda_{+}^{j}\left(\xi^{\prime}, \xi_{m}\right)}+\frac{1}{\Lambda_{+}^{k}\left(\xi^{\prime}, \xi_{m}\right)}\left(\Pi_{+} \Lambda_{+}^{k} \widetilde{u}\right)\left(\xi^{\prime}, \xi_{m}\right) .
$$


We write (4.2) in the form

$$
F_{\xi \rightarrow x}^{-1}\left(A_{\neq}(\xi) \widetilde{w}_{+}(\xi)\right)=F_{\xi \rightarrow x}^{-1}\left(B_{m} A_{=}^{-1}(\xi) \widetilde{l f}(\xi)\right)
$$

which implies

$$
\left(A_{\neq} * w_{+}\right)(x)=\chi_{C}(x) \cdot\left(A_{=}^{-1} *(l f)\right)(x),
$$

where $\chi_{C}$ is the characteristic function of the cone $C$.

Acting by the operator $\mathrm{T}_{\varphi}^{-1}$ on both sides of the last equality and passing to the Fourier images, we note that

$$
\mathrm{T}_{\varphi}^{-1}\left(\chi_{C}(x) \cdot\left(A_{=}^{-1} *(l f)\right)(x)\right)=\chi_{\mathbb{R}_{+}^{m}}(x) \cdot \mathrm{T}_{\varphi}^{-1}\left(A_{=}^{-1} *(l f)\right) .
$$

Consequently,

$$
\begin{aligned}
& \left(F T_{\varphi}^{-1}\right)\left(A_{\neq} * w_{+}\right)(\xi)=\Pi_{+} V_{\varphi}\left(A_{=}^{-1}(\xi) \widetilde{l f}(\xi)\right), \\
& \widetilde{w}_{+}(\xi)=A_{\neq}^{-1}(\xi) V_{-\varphi} \Pi_{+} V_{\varphi}\left(A_{=}^{-1}(\xi) \widetilde{l f}(\xi)\right) .
\end{aligned}
$$

Applying (4.3) to the last identity, we obtain the required expansion

$$
\widetilde{w}_{+}(\xi)=\sum_{j=1}^{n}\left[\left(A_{\neq}^{-1} \cdot V_{-\varphi} \cdot \Lambda_{+}^{-j} \cdot V_{\varphi}\right) \widetilde{v}_{j}\right]\left(\xi^{\prime}, \xi_{m}\right)+\widetilde{u}_{+}(\xi),
$$

where (cf. [10]) $\widetilde{v}_{j}\left(\xi^{\prime}\right)=i\left(\Pi^{\prime} \Lambda_{+}^{j-1} \cdot A_{=}^{-1} \cdot \widetilde{l f}\right)\left(\xi^{\prime}\right)$ and $\widetilde{u}_{+}(\xi)=A_{\neq}^{-1}(\xi) V_{-\varphi} \Lambda_{+}^{-k}\left(\Pi_{+} V_{\varphi} \Lambda_{+}^{k} A_{=}^{-1}\right.$. $\widetilde{l f})\left(\xi^{\prime}, \xi_{m}\right)$. We also extract the terms $V_{-\varphi} \Lambda_{+}^{-j} V_{\varphi} \widetilde{v}_{j} \equiv \widetilde{u}_{j}$ and write the expansion

$$
\widetilde{w}_{+}(\xi)=\sum_{j=1}^{n}\left(A_{\neq}^{-1} \cdot \widetilde{u}_{j}\right)\left(\xi^{\prime}, \xi_{m}\right)+\widetilde{u}_{+}(\xi) .
$$

The first terms in (4.4) play the role of potentials.

Theorem 4.1. Equation (4.1) with $a-s=n+\delta,-n \in \mathbb{N},|\delta|<1 / 2$, has a solution in the space $H^{s}(C)$ if and only if the right-hand side $f$ satisfies the additional conditions $\widetilde{u}_{j}=0$, $j=1, \ldots,|n|$.

\section{Acknowledgments}

The work is financially supported by the Ministry of Science and HIgher Education of the Russian Federation (project No. 1.7311.2017/8.9). The author thanks a reviewer for useful remarks.

\section{References}

1. I. M. Gel'fand and G. E. Shilov, Generalized Functions. Vol. 1: Properties and Operations, Am. Math. Soc., Providence, RI (2016).

2. I. M. Gel'fand and G. E. Shilov, Generalized Functions. Vol. 2: Spaces of Fundamental and Generalized Functions, Am. Math. Soc., Providence, RI (2016). 
3. V. S. Vladimirov, Methods of the Theory of Generalized Functions, Taylor \& Francis, London (2002).

4. V. B. Vasil'ev, "Potentials for elliptic boundary value problems in cones" [in Russian], Sib. Èlectron. Mat. Izv. 13, 1129-1149 (2016).

5. V. B. Vasil'ev, Multipliers of Fourier Integrals, Pseudodifferential Equations, Wave Factorization, and Boundary Value Problems [in Russian], URSS, Moscow (2010).

6. V. B. Vasil'ev, "On the Dirichlet and Neumann problems in multidimensional cone," Math. Bohem. 139, No. 2, 333-340 (2014).

7. V. B. Vasil'ev, Wave Factorization of Elliptic Symbols: Theory and Applications. Introduction to the Theory of Boundary Value Problems in Non-Smooth Domains, Kluwer, Dordrecht etc. (2000).

8. V. B. Vasilyev, "Pseudo-differential equations and conical potentials: 2-dimensional case," Opusc. Math. 39, No. 1, 109-124 (2019).

9. P. I. Lizorkin, "Generalized Liouville differentiation and the function spaces $L_{p}^{r}\left(E_{n}\right)$. Embedding theorems" [in Russian], Mat. Sb. 60, No. 3, 325-353 (1963).

10. G. Eskin, Elliptic Boundary Value Problems for Elliptic Pseudodifferential Equations, Am. Math. Soc., Providence RI (1981).

11. F. D. Gakhov, Boundary Value Problems, Dover, New York (1981).

12. N. I. Muskhelishvili, Singular Integral Equations, North Holland, Amsterdam (1976).

13. V. S. Vladimirov, Methods of Function Theory of Many Complex Variables [in Russian], Nauka, Moscow (1964).

14. E. M. Stein and G. Weiss, Introduction to Fourier Analysis on Euclidean Spaces, Princeton Univ. Press, Princeton (1971).

15. V. B. Vasilyev, "Pseudo-differential equations, wave factorization, and related problems," Math. Methods Appl. Sci. 41, No. 18. 9252-9263 (2018).

16. V. B. Vasilyev, "On certain elliptic problems for pseudo differential equations in a polyhedral cone," Adv. Dyn. Syst. Appl. 9, No. 2, 227-237 (2014).

17. V. B. Vasilyev, "Model elliptic boundary value problems for pseudodifferential operators in canonical nonsmooth domains," J. Math. Sci., New York 234, No. 4, 397-405 (2018).

Submitted on November 2, 2019 